\title{
ON PREDICTIVE CODING FOR ERASURE CHANNELS USING A KALMAN FRAMEWORK
}

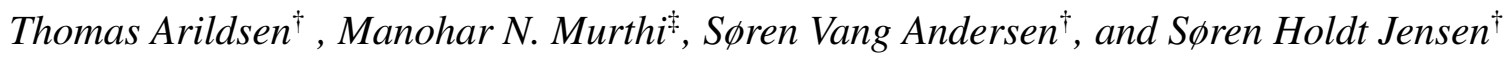 \\ $\dagger$ Dept. of Electronic Systems, Aalborg University \\ Fredrik Bajersvej 7, 9220 Aalborg, Denmark \\ phone: +45 99408684, e-mail: \{tha|sva|shj\}@es.aau.dk \\ \# Electrical and Computer Engineering, Univ. of Miami \\ 1251 Memorial Drive, Coral Gables, FL 33146, USA \\ phone: +1 305284 3342, e-mail: mmurthi@miami.edu
}

\begin{abstract}
We present a new design method for robust low-delay coding of auto-regressive (AR) sources for transmission across erasure channels. The method is based on Linear Predictive Coding (LPC) with Kalman estimation at the decoder. The method designs the encoder and decoder offline through an iterative algorithm based on minimization of the trace of the decoder state error covariance. The design method applies to stationary AR sources of any order. Simulation results show considerable performance gains, when the transmitted quantized prediction errors are subject to loss, in terms of Signal-to-Noise Ratio (SNR) compared to the same coding framework optimized for no loss. We furthermore investigate the impact on decoding performance when channel losses are correlated. We find that the method still provides substantial improvements in this case despite being designed for i.i.d. losses.
\end{abstract}

\section{INTRODUCTION}

In transmission of real-time signals data losses are typically an unavoidable impairment. Transmission can be protected against losses by, e.g., error correcting codes or Multiple Description Coding (MDC), or the effects of losses on the transmitted signal may be mitigated through various loss concealment techniques at the receiver. For low-delay coding applications, error-correcting codes are impractical due to the delay they impose. In such cases, another possibility is to modify the source coding itself to increase robustness against losses.

Linear Predictive Coding (LPC) is a principle commonly employed in speech applications. Differential Pulse Code Modulation (DPCM) is an example of a predictive source coding scheme [11]. Kalman filtering can be applied in predictive coding to provide Minimum Mean-Squared Error (MMSE) estimation of the source signal. Previous applications of Kalman filtering to predictive coding employ Kalman filters at both encoder and decoder and transmit quantized Kalman innovations from encoder to decoder, requiring synchronized encoders and decoders $[10,6,12,1]$.

When considering a Kalman filter-based decoder, the work in [12] applies to optimizing the Kalman filter for given noise statistics by selecting the optimal measurement vector that minimizes some measure on the a posteriori state error covariance. However, this approach does not take channel losses into account. Handling of lost measurements in a Kalman estimator is investigated thoroughly in $[14,13]$, but this work does not consider optimization of the coding system for such losses.

An approach for optimization of a predictive quantization scheme employing Kalman-like filters at encoder and decoder is presented in [7] where channel losses are modeled by a Markov model. [7] is contemporaneous work with a different philosophy than what we present here; it presents an optimization method based on Jump Linear System (JLS) modeling and Linear Matrix Inequal-

This work was partially financed by the Danish Research Council for Technology and Production Sciences under grant no. 274-05-0488. The work of M.N. Murthi was supported by the U.S. National Science Foundation under Awards CCF-0347229, and CNS-0519933. ity (LMI)-constrained convex optimization to design fixed gains for the encoder and decoder filters for each channel state.

In [3], we present a novel optimization method for the design of low-delay predictive coding systems, demonstrating a method for designing a robust encoder and decoder for given channel loss statistics. In particular, we examine DPCM, which is a canonical method of predictive coding which captures the basic problems of real-time transmission over channels with packet loss. In contrast to other efforts to design robust DPCM methods (e.g., [5, 7]), we consider a generalized DPCM encoder structure with separate prediction and noise feedback filters, an encoding structure commonly employed in speech coding. Moreover, we consider the case where these encoder filters are fixed time-invariant filters, leading to lowcomplexity quantization of signal samples. This encoder transmits quantization information (related to quantized prediction errors) that is subject to channel loss/erasure. The decoder views the received information from the encoder as noisy signal measurements, and utilizes Kalman filtering principles to perform Linear Minimum Mean-Squared Error (LMMSE) estimation of the signal. This approach of viewing the encoder as producing noisy measurements is in contrast to previous approaches in $[10,6,12,1]$, in which the encoder's transmitted quantized prediction error is viewed as the innovation, with both the encoder and decoder running synchronized Kalman filters.

Our predictive coding scheme consists of both offline and online stages. In the offline stage, the fixed encoder filters, and the corresponding Kalman measurement vector at the decoder are jointly designed, taking into account both the quantization noise and channel loss statistics. In the online operation, the decoder's timevarying Kalman filter estimates each signal sample, taking into account the individual loss events in the estimation. Since the encoder remains fixed while the decoder is time-varying, synchronization between encoder and decoder is not assumed. Simulation results in [3] demonstrate the efficacy of the proposed method.

In this paper we present further results investigating the performance of the proposed design method. In addition to the i.i.d. losses considered in [3], we investigate performance under Gilbert-Elliot correlated losses to assess how the method handles under more demanding loss conditions than it was intended for. We show that although overall performance of the coding framework is degraded by correlated erasures, compared to i.i.d erasures, our design method still provides significant improvements compared to the same coding framework optimized for no loss.

To illustrate the application of our design method, we employ a coding framework based on generalized DPCM coding. We chose DPCM as this captures the essence of low delay predictive coding. The principle can be extended to more complex coding schemes as well, for example based on vector quantization of LSFs (Line Spectral Frequencies) or other methods.

\section{CODING FRAMEWORK AND DESIGN METHOD}

This section describes the source encoder and decoder in Sections 2.1 and 2.2. The optimization for sample losses is treated in Section 2.3. 


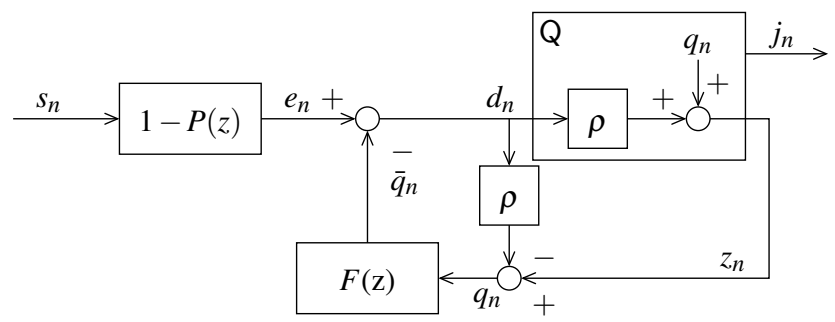

Figure 1: Generalized DPCM source encoder with Additive White Noise (AWN) quantizer model and de-correlated quantization noise feedback.

\subsection{Source Encoder}

The encoder chosen to illustrate the application of our design method is based on generalized DPCM coding. We consider an encoder with a noise shaping structure illustrated in Figure 1, [4]. The source signal and the encoder are defined by (1)-(4).

$$
\begin{aligned}
& s_{n}=\sum_{i=1}^{N} \alpha_{i} s_{n-i}+r_{n}, \\
& d_{n}=e_{n}-\bar{q}_{n}, \\
& e_{n}=s_{n}-\sum_{i=1}^{p} a_{i} s_{n-i}, \\
& \bar{q}_{n}=\sum_{i=1}^{f} b_{i} q_{n-i}
\end{aligned}
$$

The source process of order $N$ is driven by zero-mean stationary white Gaussian noise $r ; \alpha_{i}$ are the source auto-regressive (AR) coefficients, defining the source process together with $N ; e_{n}$ is the prediction error; $\bar{q}_{n}$ is the filtered quantization noise feedback; $d_{n}$ is the input to the quantizer; $q_{n}$ is the additive quantization error; $p$ is the predictor order and $a_{i}, i=1, \ldots, p$ are its coefficients; $f$ is the noise feedback filter order and $b_{i}, i=1, \ldots, f$ are its coefficients. Please note that the encoder definition allows $p \neq f \neq N$ in general.

As depicted in Figure 1, the output transmitted to the decoder is quantization indices, $j_{n}$, for the quantized prediction error, $z_{n}$, in (5), seen as the Kalman measurement by the decoder, cf. Section 2.2.

$$
z_{n}=Q\left(d_{n}\right)
$$

We use scalar quantization $Q(\cdot)$ with a gain-plus-additive-noise model [11]. The model accommodates correlation between quantizer input and quantization noise.

$$
z_{n}=\rho d_{n}+q_{n},
$$

where $\rho \in[0,1]$, and $q_{n}$ is a stationary zero-mean white Gaussian noise, independent of $d_{n}$, with variance

$$
\sigma_{q}^{2}=k \operatorname{var}\left\{d_{n}\right\}
$$

where the quantization noise is modeled with a variance proportional, by a constant $k$, to the variance of the input to the quantizer. The assumption of Gaussian $q_{n}$ is a simplifying assumption in the sense that quantization noise is generally not Gaussian and only approximately white under high-rate assumptions [9]. In order to be able to model the quantization noise as measurement noise in the Kalman filter in the decoder and to facilitate quantizer design, this noise must be white Gaussian. Since $q_{n}$ is not truly Gaussian, the decoder Kalman filter will not provide MMSE estimation of the source signal. The Kalman filter will however be the best linear estimator of the source signal-LMMSE-optimal. $\rho$ and $k$ are given by the coding loss, $\beta$, of the quantizer:

$$
\rho=1-\beta \quad k=\beta(1-\beta),
$$

where $\beta$ is the inverse of the quantizer coding gain [9].

The noise incurred by quantization is

$$
\tilde{q}_{n}=z_{n}-d_{n}=(\rho-1) d_{n}+q_{n} .
$$

In order to simplify the calculation of quantizer input variance in the optimization of the encoder, we wish to feed back a white noise component. Therefore, $d_{n}$ is scaled by $\rho$ in the quantization noise feedback to de-correlate the noise feedback from $d_{n}$. Thus, we only feed back the uncorrelated part of the quantization noise $q_{n}=z_{n}-\rho d_{n}$. This allows us to model the input to the quantizer, $d_{n}$, as Gaussian which simplifies the optimization of the encoder and the quantizer design. See [3] for design of quantizers used in the encoder.

\subsection{Kalman Filter-Based Decoder}

The decoder is based on Kalman filtering, i.e., LMMSE estimation of the source signal $s$. The Kalman filter at the decoder estimates the source signal based on measurements, $z$, reconstructed from the received quantization indices, $j_{n}$. In order to derive the Kalman estimator $\hat{s}_{n}$ of $s_{n}$, the source process and encoder equations are modeled by a state space model of the form given in, e.g., [2]. The state transition equation is chosen to represent the evolution of the source signal $s_{n}$ as well as the states of the encoder filters $P$ and $F$. The measurement equation represents the filtering and quantization operations of the encoder.

The decoder is based on the state-space model in (10) and (11).

$$
\begin{aligned}
\mathbf{x}_{n+1} & =\mathbf{F} \mathbf{x}_{n}+\mathbf{G} \mathbf{w}_{n} \\
z_{n} & =\mathbf{h}^{\mathrm{T}} \mathbf{x}_{n}+q_{n},
\end{aligned}
$$

where the state $\mathbf{x}_{n}$ is defined as

$$
\mathbf{x}_{n}=\left[\begin{array}{l|lll|lll}
s_{n} & s_{n-1} & \cdots & s_{n-p} & q_{n-1} & \cdots & q_{n-f}
\end{array}\right]^{\mathrm{T}},
$$

and the state transition matrix $\mathbf{F}$ is defined as

$$
\mathbf{F}=\left[\begin{array}{c|r|r}
\alpha_{1 \times N} & \mathbf{0}_{1 \times(p+1-N)} & \multicolumn{2}{|c}{\mathbf{0}_{(p+1) \times f}} \\
\hline \mathbf{I}_{p} & \mathbf{0}_{p \times 1} & \multicolumn{2}{|c}{\mathbf{0}_{1 \times f}} \\
\hline \multirow{2}{*}{\mathbf{0}_{f \times(p+1)}} & \frac{\mathbf{I}_{(f-1)}}{\mathbf{0}_{(f-1) \times 1}}
\end{array}\right],
$$

where $\alpha=\left[\alpha_{1} \cdots \alpha_{N}\right]$ are the coefficients of the source AR process, $\mathbf{I}_{x}$ are $x \times x$ identity matrices, and $\mathbf{0}$ are all-zero matrices (the subscripts in (13) denote the dimensions of the individual components). Thus, the top-left part of $\mathbf{F}$ represents the AR filtering of the process noise, given by (1), generating the source signal, and shifts past source signal samples through the state. The bottom-right part of $\mathbf{F}$ simply shifts previous quantization noise samples through the state. Note that this formulation allows $p \geq N-1$ and $f \geq 0$.

The process noise $\mathbf{w}_{n}$ is defined as

$$
\mathbf{w}_{n}=\left[\begin{array}{ll}
r_{n} & q_{n}
\end{array}\right]^{\mathrm{T}},
$$

which is stationary zero-mean white Gaussian with

$$
\mathbf{Q}=\operatorname{cov}\left\{\mathbf{w}_{n}, \mathbf{w}_{n}\right\}=\left[\begin{array}{cc}
\operatorname{var}\left\{r_{n}\right\} & 0 \\
0 & \operatorname{var}\left\{q_{n}\right\}
\end{array}\right]
$$

The first (scalar) component of the process noise, $r_{n}$, models the source signal excitation and the second (scalar) component, $q_{n}$, models the quantization noise fed back to the filter $F$. The definition (14) introduces correlation between the process noise $\mathbf{w}_{n}$ and the measurement noise $q_{n}$ in (11) as follows

$$
\mathbf{S}=\operatorname{cov}\left\{\mathbf{w}_{n}, q_{n}\right\}=\mathrm{E}\left\{\left[\begin{array}{l}
r_{n} \\
q_{n}
\end{array}\right] q_{n}\right\}=\left[\begin{array}{c}
0 \\
\mathbf{R}
\end{array}\right],
$$


where $\mathbf{R}=\sigma_{q}^{2}$ is a scalar since we consider scalar-valued measurements $z_{n}$. We use a formulation of the Kalman filter which takes the covariance $\mathbf{S}$ into account.

The matrix $\mathbf{G}$ is a transform to allow the process noise $\mathbf{w}_{n}$ to be defined in a compact form. $\mathbf{G}$ is given by (17).

$$
\mathbf{G}=\left[\begin{array}{cccc}
1 & \mathbf{0}_{2 \times p} & 0 & \mathbf{0}_{2 \times(f-1)}
\end{array}\right]^{\mathrm{T}}
$$

The measurement vector $\mathbf{h}$ represents the filtering operations of the encoder as well as the scaling in the model of the quantizer

$$
\mathbf{h}=\rho \tilde{\mathbf{h}},
$$

where $\tilde{\mathbf{h}}$ contains the coefficients of the prediction error and noise feedback filters

$$
\tilde{\mathbf{h}}=\left[\begin{array}{lllllll}
1 & -a_{1} & \cdots & -a_{p} & -b_{1} & \cdots & -b_{f}
\end{array}\right]^{\mathrm{T}},
$$

such that by (2),

$$
d_{n}=\tilde{\mathbf{h}}^{\mathrm{T}} \mathbf{x}_{n}
$$

whereby (11) follows from (6). To summarize, $\tilde{\mathbf{h}}$ represents the filtering in the encoder before quantization. Due to the quantization noise model presented in Section 2.1, $\mathbf{h}$ represents the filtering after quantization and produces the measurement seen by the decoder.

The decoder receives information (quantization indices $j_{n}$ ) to build measurements $z_{n}$ from the encoder.

Considering the situation where $z_{n}$ may be lost, we have a timevarying Kalman filter. The measurement vector $\mathbf{h}_{n}$ and measurement noise covariance $\mathbf{R}_{n}$ are time-varying. This models the possible loss of measurements at the decoder.

$$
\begin{aligned}
\mathbf{h}_{n} & =\gamma_{n} \mathbf{h} \\
\mathbf{R}_{n} & =\gamma_{n} \mathbf{R}+\left(1-\gamma_{n}\right) \sigma^{2} \mathbf{I},
\end{aligned}
$$

where $\gamma_{n}$ are outcomes of a random stationary Bernoulli process modeling measurement arrival with arrival probability $\operatorname{Pr}\left\{\gamma_{n}=1\right\}=$ $\bar{\gamma}$ and loss probability $\operatorname{Pr}\left\{\gamma_{n}=0\right\}=1-\bar{\gamma}$. R is the measurement noise covariance in the case of no loss and $\sigma^{2} \mathbf{I}$ is the measurement noise covariance in the case of loss. We let $\sigma^{2} \rightarrow \infty$ in (22), representing infinite uncertainty about the measurement $z_{n}$ when it is lost in transmission. See [14] for an example of this approach. Replacing $\mathbf{R}_{n}$ and $\mathbf{h}_{n}$ by (21) and (22) in the Kalman filter, as found in, e.g., [2], and taking $\lim _{\sigma \rightarrow \infty}$, we obtain

$$
\begin{aligned}
\hat{\mathbf{x}}_{n}= & \hat{\mathbf{x}}_{n}^{-}+\gamma_{n} \mathbf{P}_{n}^{-} \mathbf{h}\left(\mathbf{h}^{\mathrm{T}} \mathbf{P}_{n}^{-} \mathbf{h}+\mathbf{R}\right)^{-1}\left(z_{n}-\mathbf{h}^{\mathrm{T}} \hat{\mathbf{x}}_{n}^{-}\right) \\
\mathbf{P}_{n}= & \mathbf{P}_{n}^{-}-\gamma_{n} \mathbf{P}_{n}^{-} \mathbf{h}\left(\mathbf{h}^{\mathrm{T}} \mathbf{P}_{n}^{-} \mathbf{h}+\mathbf{R}\right)^{-1} \mathbf{h}^{\mathrm{T}} \mathbf{P}_{n}^{-} \\
\hat{\mathbf{x}}_{n+1}^{-}= & \left(\mathbf{F}-\gamma_{n} \mathbf{G} \mathbf{S} \mathbf{R}^{-1} \mathbf{h}^{\mathrm{T}}\right) \hat{\mathbf{x}}_{n}+\gamma_{n} \mathbf{G} \mathbf{S} \mathbf{R}^{-1} z_{n} \\
\mathbf{P}_{n+1}^{-}= & \left(\mathbf{F}-\gamma_{n} \mathbf{G} \mathbf{S} \mathbf{R}^{-1} \mathbf{h}^{\mathrm{T}}\right) \mathbf{P}_{n}\left(\mathbf{F}-\gamma_{n} \mathbf{G} \mathbf{S} \mathbf{R}^{-1} \mathbf{h}^{\mathrm{T}}\right)^{\mathrm{T}} \\
& +\mathbf{G}\left(\mathbf{Q}-\gamma_{n} \mathbf{S} \mathbf{R}^{-1} \mathbf{S}^{\mathrm{T}}\right) \mathbf{G}^{\mathrm{T}},
\end{aligned}
$$

The decoded signal $\hat{s}_{n}$ is the first element of $\hat{\mathbf{x}}_{n}$ according to (12). We see that the encoder takes the individual channel erasures/losses into account, represented by $\gamma_{n}=0$. This effectively reduces the current estimate of the state $\hat{\mathbf{x}}_{n}$ to the a priori estimate $\hat{\mathbf{x}}_{n}^{-}$in case of erasure of $j_{n}$, i.e., a prediction from past observations.

\subsection{Encoder and Decoder Design}

One could choose to calculate filter parameters/measurement vectors $\mathbf{h}_{n}^{*}$ at each time step $n$ to improve decoding performance at the next time step $n+1$. However, this approach would require loss-less feedback of observed arrivals $\gamma_{n}$ from decoder to encoder before time $n+1$ in order to be able to calculate identical parameters at encoder and decoder. In order not to impose this loss-less near-instantaneous feedback requirement on the system, we seek a method that allows offline calculation of a constant $\mathbf{h}^{*}$, given the statistics of loss. So, the goal is a method that improves decoding performance under average loss conditions rather than the specific loss outcomes. The offline calculation of $\mathbf{h}^{*}$ furthermore decreases the computational complexity of the framework since the calculation can be performed once in stead of being performed at each time step $n$. In contrast to Kalman filtering without loss, $\mathbf{P}_{n}$ is stochastic here due to measurement losses (the random variable $\gamma_{n}$ ). We propose the following offline method for designing measurement vectors for improved performance under sample losses. We use a simplified approach where the philosophy is to obtain $\mathbf{a} \mathbf{h}^{*}$ minimizing the expectation of $\mathbf{P}_{n}$ over current loss, $\gamma_{n}$, at time $n$.

$$
\mathbf{h}^{*}=\underset{\mathbf{h}}{\arg \min } \operatorname{Tr}\left[\mathrm{E}_{\gamma_{n}}\left\{\mathbf{P}_{n}\right\}\right]
$$

where $\mathbf{P}_{n}$ is given in (24)

Similar to [12], we express the measurement noise covarianceor equivalently, quantization noise variance- $\mathbf{R}$ as a function of $\mathbf{h}$ as given in Proposition 2.1.

Proposition 2.1.

$$
\mathbf{R}_{(i)}=\frac{k}{\rho^{2}} \mathbf{h}_{(i)}^{\mathrm{T}} \mathbf{R}_{\mathbf{x} \mathbf{x},(i)} \mathbf{h}_{(i)},
$$

where $\mathbf{R}_{\mathbf{x x},(i)}$ is the state correlation matrix, with the structure

$$
\mathbf{R}_{\mathbf{x x},(i)}=\left[\begin{array}{cc}
\mathbf{R}_{s s} & \mathbf{0} \\
\mathbf{0} & \mathbf{I}_{f} \mathbf{R}_{(i-1)}
\end{array}\right] .
$$

Proof. We refer to [3] for further details.

The index $(i)$ in Proposition 2.1 and following equations denotes the quantity calculated at iteration $i$. Using Proposition 2.1 and taking the expectation of (24) gives

$$
\mathrm{E}_{\gamma}\left\{\mathbf{P}_{(i)}\right\}=\mathrm{E}_{\gamma}\left\{\mathbf{P}_{(i)}^{-}\right\}-\bar{\gamma} \frac{\mathrm{E}_{\gamma}\left\{\mathbf{P}_{(i)}^{-}\right\} \mathbf{h h}^{\mathrm{T}} \mathrm{E}_{\gamma}\left\{\mathbf{P}_{(i)}^{-}\right\}}{\mathbf{h}^{\mathrm{T}}\left(\mathrm{E}_{\gamma}\left\{\mathbf{P}_{(i)}^{-}\right\}+\frac{k}{\rho^{2}} \mathbf{R}_{\mathbf{x} \mathbf{x},(i)}\right) \mathbf{h}},
$$

where $\bar{\gamma}$ is the arrival probability. The inverse in (24) corresponds to scalar division, because we have scalar measurements. Time index $n$ has been omitted, the index $(i)$ indicates iteration number.

$\mathbf{P}_{n}^{-}$is updated according to the discrete-time Riccati equation, [2, p. 108], of the decoder Kalman filter, adapted for measurement losses, the expectation of which is given in Proposition 2.2.

\section{Proposition 2.2.}

$$
\begin{array}{r}
\mathrm{E}_{\gamma}\left\{\mathbf{P}_{(i)}^{-}\right\}=\mathbf{F} \mathrm{E}_{\gamma}\left\{\mathbf{P}_{(i-1)}^{-}\right\} \mathbf{F}^{\mathrm{T}} \\
-\bar{\gamma} \frac{\left(\mathbf{F ~ E}\left\{\mathbf{P}_{(i-1)}^{-}\right\} \mathbf{h}+\mathbf{G S}\right)\left(\mathbf{F ~ E} \gamma\left\{\mathbf{P}_{(i-1)}^{-}\right\} \mathbf{h}+\mathbf{G S}\right)^{\mathrm{T}}}{\mathbf{h}^{\mathrm{T}}\left(\mathrm{E}_{\gamma}\left\{\mathbf{P}_{(i-1)}^{-}\right\}+\frac{k}{\rho^{2}} \mathbf{R}_{\mathbf{x x},(i)}\right) \mathbf{h}}+\mathbf{G Q G}^{\mathrm{T}} .
\end{array}
$$

Proof. We refer to [3] for further details.

This requires the arrival probability $\bar{\gamma}$ to be known in order to design the encoder and decoder.

At each iteration $i, \mathbf{h}_{(i)}$ is selected to minimize the trace of (30) according to (32).

$$
\mathbf{h}_{(i)}=\underset{\mathbf{h}}{\arg \max } \frac{\mathbf{h}^{\mathrm{T}}\left(\mathrm{E}_{\gamma}\left\{\mathbf{P}_{(i)}^{-}\right\}\right)^{2} \mathbf{h}}{\mathbf{h}^{\mathrm{T}}\left(\mathrm{E}_{\gamma}\left\{\mathbf{P}_{(i)}^{-}\right\}+\frac{k}{\rho^{2}} \mathbf{R}_{\mathbf{x x},(i)}\right) \mathbf{h}},
$$




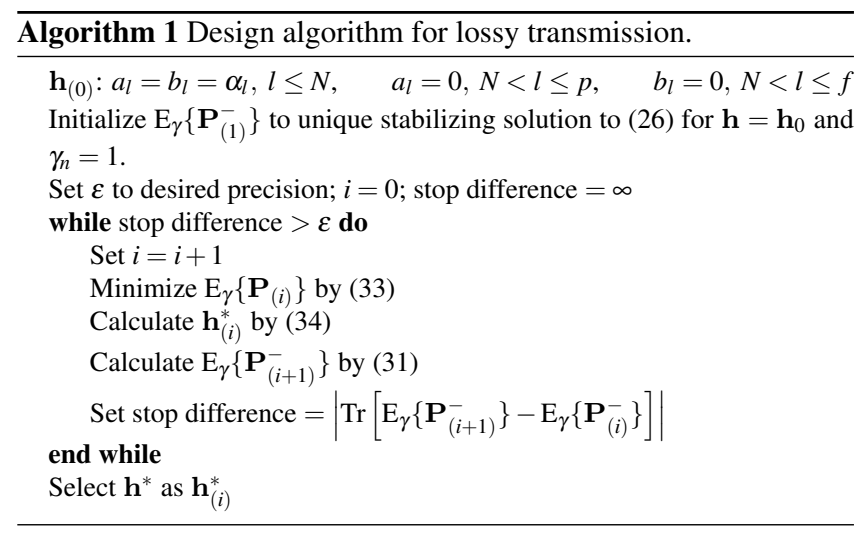

in which $\left(\mathrm{E}_{\gamma}\left\{\mathbf{P}_{(i)}^{-}\right\}\right)^{2}$ is short for $\mathrm{E}_{\gamma}\left\{\mathbf{P}_{(i)}^{-}\right\}^{\mathrm{T}} \mathrm{E}_{\gamma}\left\{\mathbf{P}_{(i)}^{-}\right\}$since $\mathbf{P}_{(i)}^{-}$is symmetric.

Equation (32) may be rewritten as a Rayleigh quotient through a Cholesky factorization $\mathbf{L} \mathbf{L}^{\mathrm{T}}=\mathrm{E}_{\gamma}\left\{\mathbf{P}_{(i)}^{-}\right\}+\frac{k}{\rho^{2}} \mathbf{R}_{\mathbf{x} \mathbf{x},(i)}$ where $\mathbf{L}$ is a lower triangular matrix. This allows us to obtain $\mathbf{h}_{(i)}$ as outlined in Proposition 2.3.

\section{Proposition 2.3.}

$$
\mathbf{y}_{(i)}^{*}=\underset{\mathbf{y}}{\arg \max } \frac{\mathbf{y}^{\mathrm{T}} \mathbf{L}^{-1}\left(\mathrm{E}_{\gamma}\left\{\mathbf{P}_{(i)}^{-}\right\}\right)^{2} \mathbf{L}^{-\mathrm{T}} \mathbf{y}}{\mathbf{y}^{\mathrm{T}} \mathbf{y}}
$$

is given as the eigenvector of $\mathbf{L}^{-1}\left(\mathrm{E}_{\gamma}\left\{\mathbf{P}_{(i)}^{-}\right\}\right)^{2} \mathbf{L}^{-\mathrm{T}}$ corresponding to its largest eigenvalue [12]. We obtain the measurement vector from $\mathbf{y}_{(i)}^{*}$ given by (33) with a normalization by the first element of the vector in order to keep $\mathbf{h}_{(i)}$ as formulated in (19), with its first element equal to 1 .

$$
\tilde{\mathbf{h}}_{(i)}=\frac{\mathbf{L}^{-\mathrm{T}} \mathbf{y}_{(i)}^{*}}{c} \quad \mathbf{h}_{(i)}=\rho \tilde{\mathbf{h}}_{(i)},
$$

where $c$ is the first element of the vector $\mathbf{L}^{-\mathrm{T}} \mathbf{y}_{(i)}^{*}$.

Proof. We refer to [3] for further details.

Equations (33), (34) and (31) are iterated until convergence of (31), upon which the resulting $\mathbf{h}_{(i)}$ is chosen as fixed measurement vector $\mathbf{h}^{*}$ for the decoder given by (23)-(26) and the corresponding $\tilde{\mathbf{h}}^{*}$ for the encoder given by the relation (18). The design method is summarized in Algorithm 1.

\section{SIMULATIONS}

In [3] we present examples of decoded Signal-to-Noise Ratio (SNR) performance gains achieved by the design presented in Section 2.3. In this paper we present additional simulation results for a source process with a somewhat different power spectral shape than the examples in [3].

- A stationary random source signal was generated from an AR(10) process. The source signal was encoded with encoder and decoder designed for each simulated arrival probability $\bar{\gamma}$. The quantization indices with losses were decoded using the Kalman decoder given by (23)-(26). This setup is referred to as "Iterative Measurement Vector Improvement (IMVI)".

- As a baseline for comparison, the same source signal was encoded and decoded with encoder and decoder designed for no loss $(\bar{\gamma}=0)$. We shall denote this baseline method "Baseline".

Sample arrivals $\gamma_{n}$ were simulated as:
- Independent identically distributed arrivals, modeled as outcomes of a Bernoulli random process over a series of arrival probabilities $\bar{\gamma} \in[0,1]$ and applied to the transmitted encoder quantization indices $j_{n}$. This loss process is referred to as "i.i.d" in the following.

- Outcomes of a Gilbert-Elliot loss process simulated as a twostate Markov process where state 1 corresponds to loss $\left(\gamma_{n}=\right.$ $0)$ and state 2 to correct arrival $\left(\gamma_{n}=1\right)$. The state transition probabilities are

$$
\begin{aligned}
& p_{1,1}=1-p_{1,2}=\lambda_{1} \\
& p_{2,1}=1-p_{2,2}=\lambda_{2} .
\end{aligned}
$$

The loss model will remain in the loss state for exponentially distributed times with mean error burst length $1 /\left(1-\lambda_{1}\right)$. The stationary distribution of the Markov chain is

$$
q_{1}=1-q_{2}=\frac{\lambda_{2}}{1-\lambda_{1}+\lambda_{2}},
$$

where $q_{2}$, the probability of being in the no-loss state, corresponds to the overall arrival probability $\bar{\gamma}$ in the filter design algorithm of Section 2.3. We refer to loss processes of this type as "GE-2" and "GE-3" for mean error burst lengths of 2 and 3, respectively.

Algorithm 1 is designed for i.i.d. losses only, but we include correlated losses in the simulations to assess the algorithm's performance under more difficult loss conditions. The simulations have been conducted for a Lloyd-Max quantizer at 2 bits/sample.

Test data were generated from an AR model estimated from a $20 \mathrm{~ms}$ sub-sequence selected from a voice-active region of speech found in [8]. The coefficients used were: $\alpha_{1}=0.8694, \alpha_{2}=$ $-0.4616, \alpha_{3}=0.0186, \alpha_{4}=0.4603, \alpha_{5}=-0.2236, \alpha_{6}=0.0302$, $\alpha_{7}=0.1843, \alpha_{8}=-0.1721, \alpha_{9}=0.0239, \alpha_{10}=-0.2115$. The power spectrum of the source AR process is plotted in Figure 2. Decoded signal SNR is compared for IMVI and Baseline in Figure 3 for a range of loss probabilities.

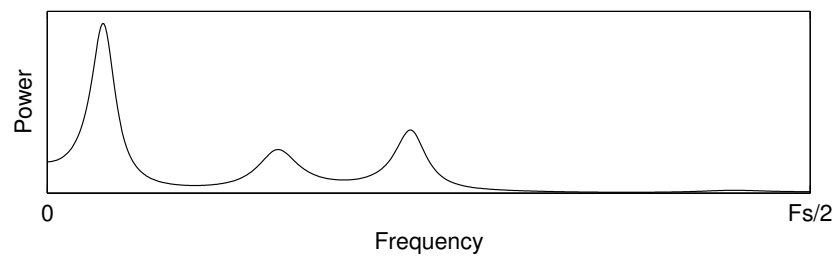

Figure 2: Power spectral density of 10th order AR source signal.

Figure 3 first and foremost shows how IMVI is capable of improving decoded signal SNR over the coding framework, Baseline, which has not been optimized for loss. In all three loss scenarios, IMVI improves SNR by, at best, $1.1 \mathrm{~dB}$ to $2.0 \mathrm{~dB}$ with most notable improvements above $5 \%$ loss rate. The plots show that the improvement obtained by our method is degraded as the losses become correlated; in the i.i.d. loss scenario, we observe improvements of up to $2.0 \mathrm{~dB}$ (appr. $23 \%$ loss rate); in the GE-2 scenario, we observe improvements of up to $1.5 \mathrm{~dB}$ (appr. $34 \%$ loss rate); in the GE-3 scenario, we observe improvements of up to $1.1 \mathrm{~dB}$ (appr. $34 \%$ to $48 \%$ loss rate). This indicates that the design algorithm is still effective at correlated loss scenarios, although not intended for such losses, but performance improvements are more modest in these cases.

\subsection{Parameter Validation}

We evaluate the correspondence between the expected state error covariance $\mathrm{E}_{\gamma_{n}}\left\{\mathbf{P}_{n}\right\}$ used in (27) and the empirical average over actual observed $\mathbf{P}_{n}$ in the running decoder, denoted avg $\left(\mathbf{P}_{n}\right)$. This is done to validate that the optimization objective used in Algorithm 1 is reasonable. 


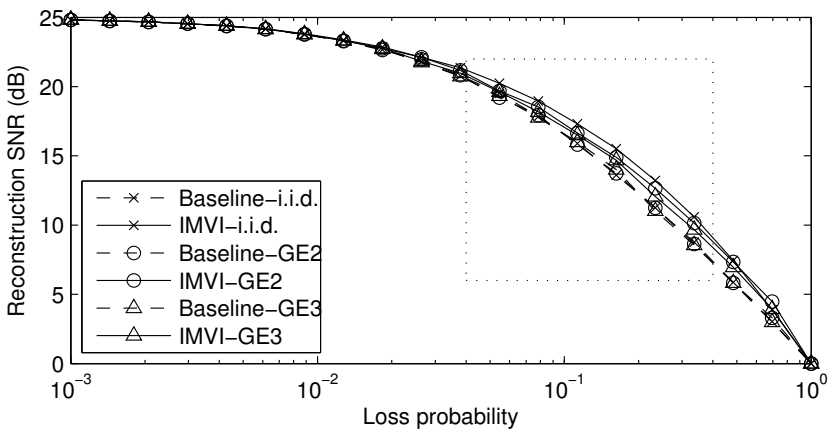

(a)

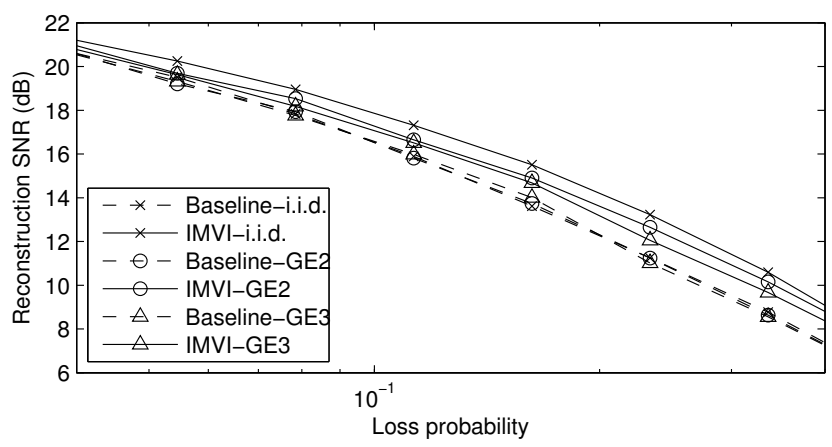

(b)

Figure 3: Decoded signal SNR for 10th order AR source signal. Plot (b) shows the marked segment of plot (a) enlarged.

We investigate $\operatorname{Tr}\left[\mathbf{P}_{n}\right]$ since the diagonal elements are the error variances of the estimated state elements. These relate directly to the SNR of the decoded signal. The resulting $\operatorname{Tr}\left[\mathrm{E}_{\gamma_{n}}\left\{\mathbf{P}_{n}\right\}\right]$ from Algorithm 1 is compared to $\operatorname{Tr}\left[\operatorname{avg}\left(\mathbf{P}_{n}\right)\right]$ observed at the decoder and averaged over $10^{5}$ samples. We compared the measured quantities at a loss rate of $1-\bar{\gamma}=0.2336$ :

$$
\begin{aligned}
\operatorname{Tr}\left[\mathrm{E}_{\gamma_{n}}\left\{\mathbf{P}_{n}\right\}\right] & =7.3266 \\
\operatorname{Tr}\left[\operatorname{avg}\left(\mathbf{P}_{n}\right)\right]_{\text {i.i.d. }} & =6.7400 \\
\operatorname{Tr}\left[\operatorname{avg}\left(\mathbf{P}_{n}\right)\right]_{\text {GE-2 }} & =7.5081 \\
\operatorname{Tr}\left[\operatorname{avg}\left(\mathbf{P}_{n}\right)\right]_{\text {GE-3 }} & =7.9330
\end{aligned}
$$

From (38) and (39) we observe that Algorithm 1 seems to overestimate the state error covariance in this example. From (38), (40) and (41) it is observed that Algorithm 1 apparently under-estimates the state error covariance in the case of correlated losses in this example. This could explain the smaller improvement obtained by the algorithm under correlated losses. At the same time it seems that over-estimating the state error covariance (the i.i.d. case) does not have as large an impact on the resulting improvement.

\section{CONCLUSION}

We have presented a novel method for optimization of predictive quantization of AR signals for transmission across erasure channels. An important contribution of the presented method is a coding framework "design philosophy" that considers the encoding a process that produces noisy measurements of the source signal. The decoding is viewed as optimal linear estimation of the source signal based on these measurements.

The proposed method provides offline design of the encoder and decoder. By taking channel erasures into account in minimizing the trace of the Kalman state error covariance, we have obtained a design method that allows selection of encoder and decoder parameters which improve robustness to losses in a framework that provides LMMSE estimation given the received measurements at the decoder.

We point out that the presented design method can be applied to predictive coding systems in general and is not limited to the particular framework presented in this paper.

The presented method has been demonstrated to improve decoded signal SNR substantially under sample erasure conditions for a diverse selection of source signal models [3]. In this paper, we present results for another signal model in addition to the examples in [3] and for correlated channel erasures. These results show that the presented method is capable of improving decoded signal quality substantially under loss conditions when losses become correlated, despite the fact that the method is designed for i.i.d. losses.

In the presented example, the design method calculates a larger expected state error covariance than what is observed in the actual decoder; i.e. the encoder and decoder are designed somewhat conservatively, corresponding to a slightly higher loss rate than the actual rate. This indicates room for additional improvements if this behaviour generalizes to other signal models.

\section{REFERENCES}

[1] S. V. Andersen, S. H. Jensen, and E. Hansen. Quantization noise modeling in low-delay speech coding. In Proc. IEEE Workshop on Speech Coding For Telecommunications Proceedings, pages 65-66. IEEE, 1997.

[2] B. D. O. Anderson and J. B. Moore. Optimal Filtering. Dover, New York, 2005.

[3] T. Arildsen, M. N. Murthi, S. V. Andersen, and S. H. Jensen. On predictive coding for erasure channels using a kalman framework. IEEE Trans. Signal Process. Accepted for publication. Available at: http://es.aau.dk/staff/tha/ publications/Arildsen2009.pdf.

[4] B. Atal and M. Schroeder. Predictive coding of speech signals and subjective error criteria. IEEE Trans. Acoust., Speech, Signal Process., 27(3):247-254, 1979.

[5] M. H. Chan. The performance of DPCM operating on lossy channels with memory. IEEE Trans. Commun., 43(234):1686-1696, Feb-Mar-Apr 1995.

[6] S. Crisafulli, J. D. Mills, and R. R. Bitmead. Kalman filtering techniques in speech coding. In 1992 IEEE International Conference on Acoustics, Speech, and Signal Processing, volume 1, pages 77-80 vol.1, 1992.

[7] A. K. Fletcher, S. Rangan, V. K. Goyal, and K. Ramchandran. Robust predictive quantization: Analysis and design via convex optimization. IEEE J. Sel. Top. Sign. Proces., 1(4):618632, Dec. 2007.

[8] J. S. Garofolo. DARPA TIMIT acoustic-phonetic speech database training set, CD-ROM prototype distribution.

[9] A. Gersho and R. M. Gray. Vector Quantization and Signal Compression. Kluwer Academic, Norwell, MA, 1992.

[10] J. Gibson, S. Jones, and J. Melsa. Sequentially adaptive prediction and coding of speech signals. IEEE Trans. Commun., 22(11):1789-1797, Nov. 1974.

[11] N. S. Jayant and P. Noll. Digital Coding of Waveforms: Principles and Applications to Speech and Video. Prentice-Hall, Englewood Cliffs, NJ, 1984.

[12] T. V. Ramabadran and D. Sinha. Speech data compression through sparse coding of innovations. IEEE Trans. Speech Audio Process., 2(2):274-284, Apr. 1994.

[13] L. Schenato, B. Sinopoli, M. Franceschetti, K. Poolla, and S. S. Sastry. Foundations of control and estimation over lossy networks. Proc. IEEE, 95(1):163-187, Jan. 2007.

[14] B. Sinopoli, L. Schenato, M. Franceschetti, K. Poolla, M. I. Jordan, and S. S. Sastry. Kalman filtering with intermittent observations. IEEE Trans. Automat. Contr., 49(9):1453-1464, Sept. 2004. 